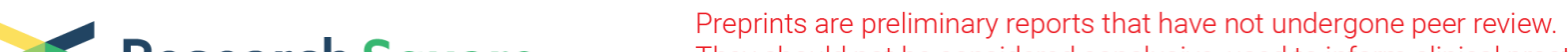 Research Square They should not be considered conclusive, used to inform clinical practice, or referenced by the media as validated information.
}

\section{An Experimental Evaluation of Hybrid Learning Methodology based Internet of Things Assisted Health Care Monitoring System}

\section{Kamal Upreti}

Dr Akhilesh Das Gupta Institute of Technology and Management

MAHAVEERAKANNAN R ( $\square$ mahaveerakannan10@gmail.com )

Hindusthan College of Engineering and Technology

Raut Ranjana Dinkar

Sant Gadge Baba Amravati University

Sudhanshu Maurya

Graphic Era Hill University

Venkatramanan Reddy

Anurag University

Thangadurai $\mathbf{N}$

Sankalchand Patel University

\section{Research Article}

Keywords: Healthcare, Deviated Learning-based Health Analysis, DLHA, Smart Health Indicator, SHI, Internet of Things, loT, Deep Learning

Posted Date: November 16th, 2021

DOl: https://doi.org/10.21203/rs.3.rs-918311/v1

License: (c) (1) This work is licensed under a Creative Commons Attribution 4.0 International License. Read Full License 


\title{
An Experimental Evaluation of Hybrid Learning Methodology based Internet of Things Assisted Health Care Monitoring System
}

${ }^{1}$ Dr Kamal Upreti, ${ }^{2}$ Dr Mahaveerakannan R, ${ }^{3}$ Dr Raut Ranjana Dinkar, ${ }^{4}$ Dr Sudhanshu Maurya, ${ }^{5}$ Dr Y Venkataramanan Reddy and ${ }^{6}$ Dr. Thangadurai $\mathrm{N}$

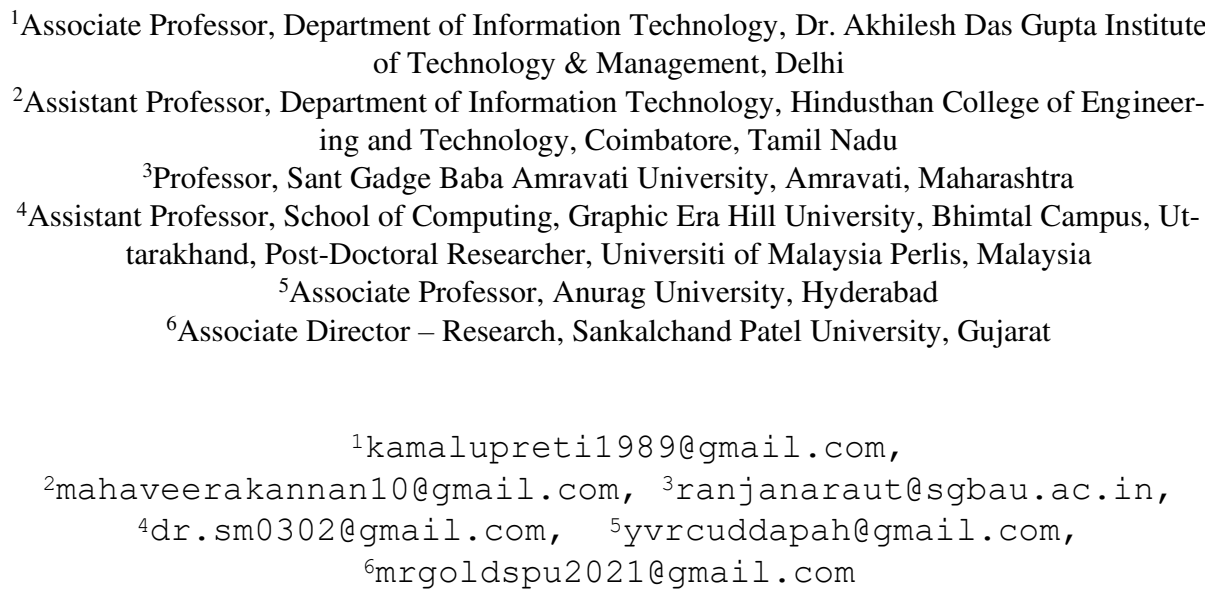

\begin{abstract}
In this modern world, every individual uses intelligent devices to lead a day-to-day activity intelligently. Using the latest technologies such as deep learning, the Internet of Things (IoT) forth provides standard prediction and communication abilities to the existing applications to properly provide rich support to the clients. Many commercial and non-commercial organizations almost adapt these technologies to modify their physical records digitally. This paper designed a novel health care monitoring scheme by adapting these technologies to provide an intelligent monitoring system to analyze patients over random instances with periodic intervals. This paper introduced a new learning-based scheme called Deviated Learning-based Health Analysis (DLHA), in which it combines the conventional algorithms such as Convolutional Neural Network (CNN) and the Support Vector Classification (SVM) logic in a transparent manner. The logical evaluations of the proposed approach called DLHA assessed by extracting the layers from the CNN, appending the classification logic of SVM into the CNN layers, and defining a new algorithm to predict patient health intelligently. The association of sensor-based smart device called Smart Health Indicator (SHI) provides significant support to the proposed approach with the association of intelligent sensors such as Heartbeat Analyzer, Body Temperature Estimation Sensor, Breath Sensor, Global Positioning System (GPS), and the useful Internet of Things enabled controller called ESP8266. Using this SHI kit, the patient details
\end{abstract}


are monitoring instantly and reporting it to the remote server periodically to analyze the health summary without any interventions. The proposed deep learning strategy called DLHA acquires the data from the intelligent health care kit SHI and processes it using classification principles. The records collected from the kit were manipulated according to the process of the trained model generated from the previous testing samples of the patients. The dataset used in this system is generated dynamically from the real-time patient health record and processes the testing report of the patient accordingly. The processed record is appended into the dataset for further reference. The resulting section provides proper proof of the efficiency of the proposed approach in a transparent manner with graphical representations. For all this system is more significant to identify and monitor the health details of the patient in clear manner with proper specifications.

Keywords: Healthcare, Deviated Learning-based Health Analysis, DLHA, Smart Health Indicator, SHI, Internet of Things, IoT, Deep Learning

\section{INTRODUCTION}

Nowadays, the consciousness of healthcare and the associated fields are rising drastically to people. The growth of the human population is increasing every day. Such changes require modern assistance to monitor the health-related details and summary instantly without any hurdles. This approach provides a mindset to clients to acquire an intelligent system to monitor health-related details from anywhere by using intelligent mobiles, PCs, tablets, and laptops. This approach is helpful to people such as students studying abroad, and their parents are in local as well as caretakers who are constantly monitoring the patients periodically. This intelligent system provides an ability for the caretakers to monitor the patient's or elder people's health records from anywhere in the world at any time without any restrictions. This system is highly required because the people cause sudden affection due to cardiac-oriented problems and other severe issues in the unknown period, in which the caretakers or doctors are unaware of such problems over an instance. But this cause creates a massive loss to patient lives and creates several complexities to patients - a new health care monitoring system with the adaptation of several latest technologies introduced in this paper. The latest technologies associated with this system are the Internet of Things, Smart Health Indicator, and the Hybrid Deep Learning scheme called Deviated Learning-based Health Analysis. By using these technologies, a powerful and robust health monitoring scheme is introduced in this paper.

\subsection{Motivation}

In the country of India, according to the "Hindustan-Times" on 2017 September-30, the 10 leading causes of death are cardiovascular disease [1], diarrhoea, respiratory illnesses, brain hemorrhage, premature births, obstructive pulmonary disease, road deaths, Perinatal infections, and infectious diseases such as tuberculosis [2]. A few of these causes of mortality, such as cardiovascular disease, strokes, newborn fatalities, 
Perinatal losses [3][4], and lower respiratory infections, can be eliminated or decreased. This is now achievable because of the proliferation of the Internet of Things and the associated learning-based prediction technologies. For example, numerous diseases are unanticipated and aggressive, including cardiovascular events. Whenever someone has a massive heart attack unexpectedly, quick action must be taken to preserve the individual's life. With the Internet of Things model reemerging, it can measure a variety of critical human health characteristics. The obtained Heartbeat can then be communicated to specialists in remote areas at a low cost and instantaneously. The respective physicians can then advise a relevant life-preserving strategy as a response.

\subsection{Healthcare with IoT}

The Internet of Things (IoT) is a general term that refers to a network interconnected and may be retrieved from any location [5]. In addition, the IoT is a term that refers to portable systems connected to the internet and communicate with one another [6]. The Internet of Things systems utilizes a wide range of sensors and controllers to render the solution intelligent. Since novel emergent innovations evolve, the Internet of Things' significance to health care is indeed critical. Therefore, mechanization is required in all fields in the coming future.

\section{Present Health care Development and Provisions}

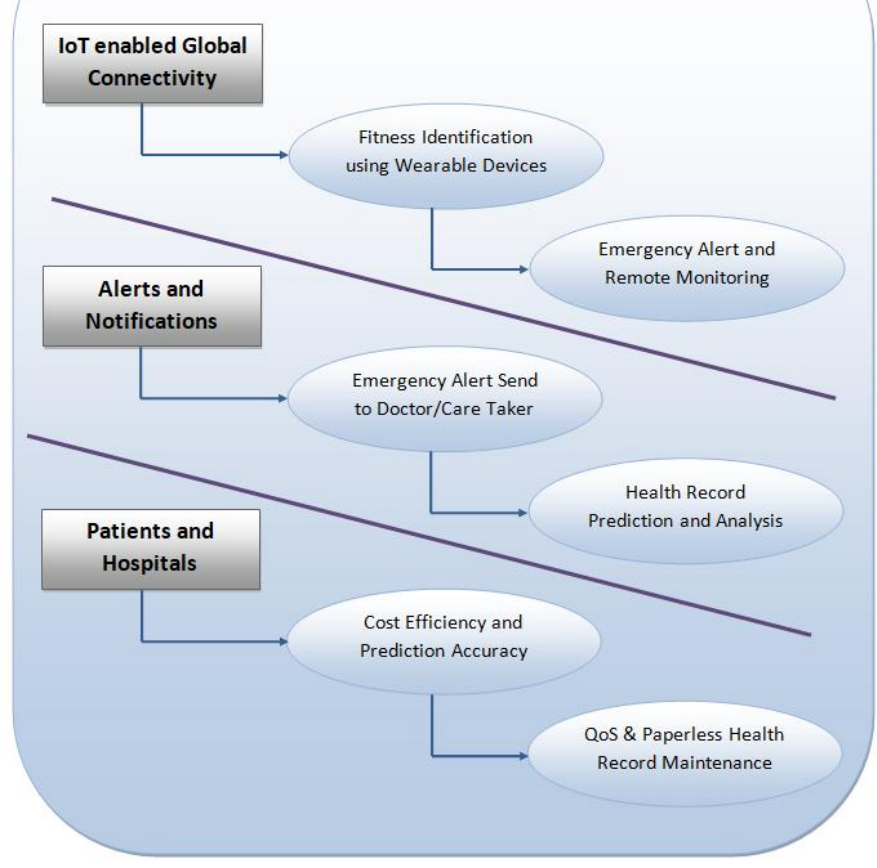

Fig. 1. Healthcare Systems - Provisions and Development 
The Internet of Things offers superior services in various domains, including green infrastructure, heavy transportation, wastewater treatment, systemic health care, communications, protection, control systems, rescue services, and medical services [7]. Health care is a prime example of an IoT deployment, in which it enables effective services for a variety of medical procedures, including chronic illnesses, exercise programs, and patient monitoring.

As a result, numerous elements utilized in medical product manufacturers, such as sensors, controllers, and computer vision equipment, are developed, using the concept of the Internet of Things as a core element of the structure. Expense-free and reliable contacts between patient characteristics and multiple hospitals are presently prominent developments in the health care profession. Numerous wireless devices are critical in assisting in the treatment of a variety of illnesses and healthcare crises. Based on various logistical considerations, the healthcare industry has become a focal point for discussing many facets of the Internet of Things over the previous decade. The following figure, Fig-1 [8], illustrates current health care developments.

\subsection{Major Objectives}

The following are the primary objectives of the proposed hybrid learning-based health care monitoring system, in which these objectives are derived for the precise analysis of literature mentioned in this paper section, Section-II.

(i) To assist the health care industry with modern technologies to preserve individual's life properly.

(ii) To introduce new hybrid health care prediction logic with the association of deep learning methodologies such as CNN and SVM to provide significant support to the patients and hospital end.

(iii) To introduce an intelligent health monitoring device with the adaptation of several sensors to monitor the patient details continuously without any human interventions and maintain the details without any human flaws.

(iv) To adapt the latest technology called the Internet of Things to preserve the health records into the remote server for maintaining it in a secured manner.

(v) To provide a good health summary to the patients and caretakers to monitor the patient details instantly without any range restrictions, in which it provides clean and transparent services to people in a clear way.

These objectives are accomplished by integrating the learning technology with the intelligent Internet of Things enabled logic to define a new Deviated Learning-based Health Analysis (DLHA) algorithm. It provides an ability to the proposed approach to build a robust health summary investigation and provides proper predictions accordingly. Furthermore, the Smart Health Indicator (SHI) kit is introduced to monitor the patient records in a 24/7 manner and report the details to the controller for maintaining it into the remote server and portraying it to the respective individuals without any delay. By applying this kind of logic, the proposed approach achieves a significant way 
to accomplish the objectives, and the resulting section provides the proper proof for that transparently.

The reminder sections of this paper provide a complete description of Related Study in Section-II, Methodologies in Section-III, Results, and Discussions in Section-IV, and the Conclusion and Future Scope transparently portrayed over Section-V.

\section{Related Study}

Deepak-Kumar et al., 2019 [11] suggested a paper regards enhancing the health care monitoring System using the Internet of Things framework. This paper [11] suggests that the Internet of Things is a cutting-edge technology facilitating a paradigm change in ancient healthcare practices. With the introduction of advanced accurate sensors and Internet of Things-enabled medicinal gadgets, human services can gradually be decentralized from the traditional healthcare focus-based methodology. These minor structure factor IoT human services devices have decreased the cost of pharmaceutical services, resulting in greater affordability and choices. This article discusses recent advancements in the engineering and framework structure of IoT-based medical service frameworks. Frameworks for medical services such as m Health and 6LoWPAN-based designs can be examined. Multi-sensor architecture designs that detect serum glucose, wireless temperature, pulse, and electrocardiogram heart are investigated. The study provides an in-depth examination of recent advancements in IoT-based human services frameworks.

Ibrahim et al., 2019 [12] suggested a paper regards the secured Edge-of-Things (EoT) framework for Intelligent health care monitoring. This paper [12] suggests that the exponential growth of the IoT and cloud-based data processing technologies enables the development of unique and exciting innovative, linked healthcare systems. Intelligent healthcare systems analyze IoT-generated patient data to improve the quality of care while also lowering healthcare expenses. However, the Cloud of Things' ability to manage the data created by billions of linked IoT devices is a significant problem for these systems. Edge computing infrastructure offers a possible alternative by acting as a bridge between IoT devices and cloud computing. The Edge of Things (EoT) can provide small-scale real-time computing and storage capabilities that assure minimal latency and optimal exploitation of the Internet of Things (IoT) resources. The EoT, on the other hand, poses privacy concerns, which is a significant problem for healthcare systems that include sensitive patient data. This article introduces a revolutionary Internet of Things computing platform for delivering safe and intelligent healthcare surveillance services. Fully Homomorphic encryption protects data privacy and is stored and processed within an EoT framework. For the proposed EoT framework, a distributed approach based on clustering-based approaches is created with the scalability to gather and analyze large-scale and heterogeneous data in distributed EoT devices independently before it is delivered to the cloud. We show the suggested framework by 
examining individual bio-signal data in a case study. Our framework significantly reduces the time required for analysis response and performance of encrypted data processing while maintaining a high level of analytical accuracy and data privacy.

\section{Methodologies}

This paper introduced a new deep learning algorithm called Deviated Learning-based Health Analysis (DLHA), in which it monitors the patient details with the help of an intelligent sensor-associated device called Smart Health Indicator. The SHI consists of several intelligent sensors to monitor the respective patient details properly, which contains the sensors such as Heartbeat Monitoring Sensor, Body Temperature Identification Sensor, Breath Level Indicator, GPS Module, and the ESP8266 controller. The proposed SHI kit accumulates the patients' health record and sends to the microcontroller for further processing by associating all these sensors. The controller associated with SHI called ESP8266 inbuilt contains WiFi unit and processing abilities, in which it accumulates the live data and separates it by using the Comma Separator Value (CSV). The separated values are sent to the remote server through internet-enabled services using the Internet of Things feature. The server unit processing machine accumulates the details sent by the controller to evaluate the data using a learning approach called DLHA. The values received into the server are indexed appropriately using the CSV application, and the data are cross-validated with the threshold level mentioned in the trained model. The present data received from the SHI is considered to be the testing data, in which it is cross-validated, and the respective indications are sent to the storage unit. The data, which consists of abnormal medical content, indicates the patient is abnormal and needs further immediate treatment. The corresponding notification is sent to the doctor and the caretaker to take appropriate actions to save the patient's life. The following summary indicates the purpose of using these sensors associated with the SHI.

\subsection{Heartbeat Monitoring Sensor}

There has been a significant rise in cardiovascular disorders, most of which result in cardiac arrest. Heartbeat monitoring sensor-assisted systems based on the Internet of Things is utilized to determine the patient's heart rate via heartbeats. This does not seem to be performed at the hospitals; this can be conducted at the person's home. The Heartbeat monitoring sensor is interconnected to an appropriate ESP8266 microcontroller, which monitors heartbeats and communicates data through the internet. The respective patient can define the heart rate's upper and lower limits on the Internet of Thingsenabled heartbeat monitoring system. Even after defining the heartbeat restrictions, an alert is issued for the controller whenever the level exceeds a predetermined threshold, indicating the physician is online. It also permits the presentation of the person's actual heartbeat rate. As a result of these features, doctors are aware of any emergencies and can swiftly contact the patient for resolution. In this system, a novel heartbeat sensor 
called Pulse Rate Amped is used to monitor the patients' heartbeat rate, which is graphically illustrated over the following figure, Fig-2.

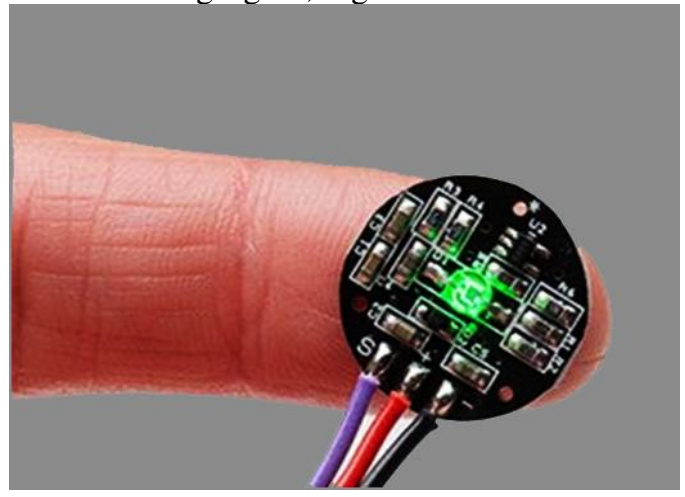

Fig. 2. Pulse Rate Amped Sensor

\subsection{Body Temperature Identification Sensor}

This body temperature is a critical characteristic for protection and wellness, and the "National Institute of Clinical Excellence" recommends that people have their temperature taken as part of their disease evaluation. There are numerous methods for determining one's body temperature. It is critical to utilize an adequate method for measuring body temperature to obtain an exact reading, as erroneous readings may result in inaccurate therapy and diagnosis, resulting in an inability to determine the actual condition. In this system, a novel DS18B20 temperature identification sensor is utilized to continuously monitor the body temperature level of the patients, which is graphically illustrated over the following figure, Fig-3. The accumulated temperature readings are sent to the controller for evaluation and passed to the server unit for further analysis; if the temperature level exceeds the average level, they are immediately indicated to the respective individual to take appropriate action.

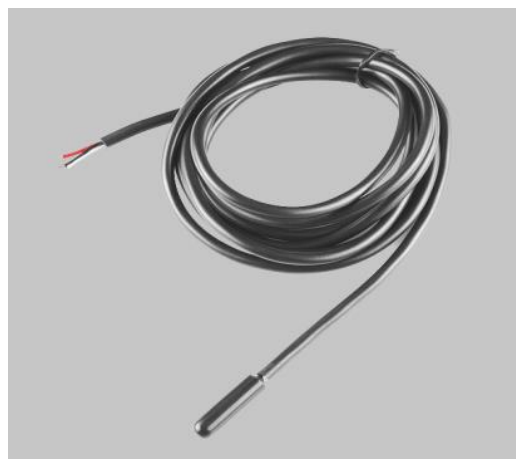

Fig. 3. DS18B20 Sensor 


\subsection{Breath Level Indicator}

In respiratory systems, slightly elevated sensors identify tiny volume flow rate from around breathing flow over 0 points and mass flow percentage with several hundred liters per minute. Types of sensors regularly review the treatment level in regulated continuous positive airway pressure systems, enhancing patient convenience and medication effectiveness. Breathing induction sonography measures variations in breadth throughout breathing by connecting two capacitive straps across the abdominal region and ribs. Calculating the breathing amount requires knowledge of the difference in perimeter, including both places. High-quality sensors integrated into breathing devices are designed to measure the tiniest flow rates near the breathing levels over starting state. In this system, a novel breath rate estimation sensor is used, and the graphical illustration of the sensor is mentioned in the following figure, Fig-4.

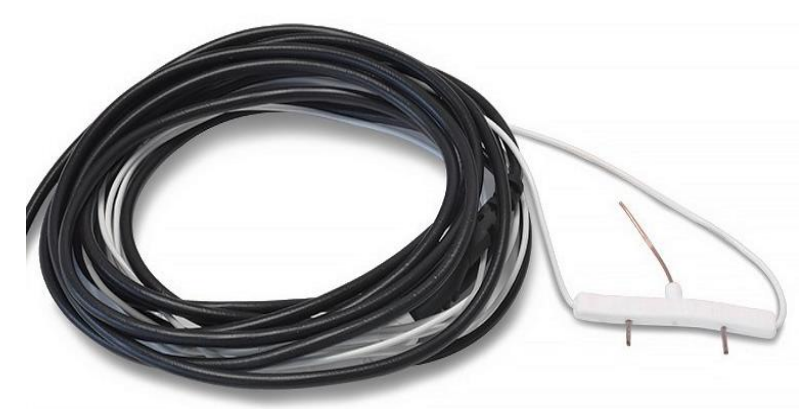

Fig. 4. Breathe Sensor

\subsection{GPS Module}

GPS modules are comprised of minuscule CPUs and antennas that acquire the information directly from the satellite via specific radio transmissions. It receives timestamps and other information from every accessible plane. The device's GPS receiver monitors all of these signals

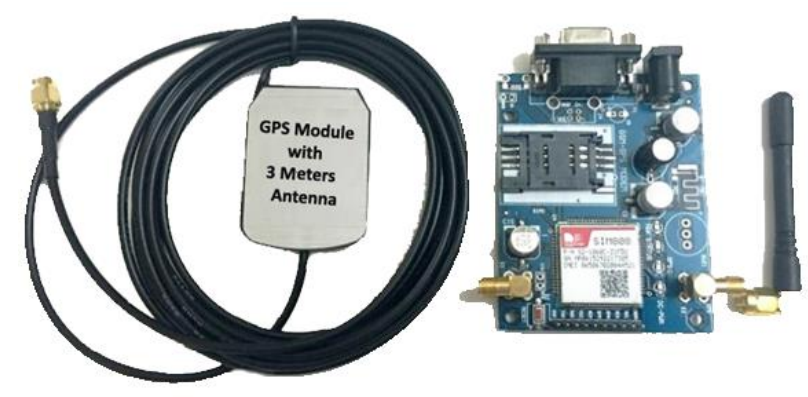

Fig. 5. GPS Module 
Once the transmitter determines its range from three or even more geostationary satellites, it's indeed capable of determining actual location. GPS devices are receivers equipped with antennas that use a satellite-based navigation system comprised of 24 orbiting satellites around the planet to deliver location, acceleration, and time data. This system is composed of three portions: environment, controls, and users. The global positioning system is accessible for everyone around the world. That is why the GPS can function even if the infotainment system does not have internet access. In this system, a novel three-meter antenna-assisted GPS module is used, and the graphical illustration of the utilized GPS module is mentioned in the following figure, Fig-5.

\subsection{ESP8266 Controller}

The ESP8266 module is a highly Wi-Fi-enabled microcontroller from Espressif Technologies in China (Shanghai City). It includes a complete Protocol of TCP/IP stack and a microcontroller. These microcontrollers were superseded by the ESP32 series of devices, which includes the ESP32 C3 version. The ESP8266 feature allows embedded systems to communicate to IEEE-802.11 standard with $\mathrm{w}-2.4 \mathrm{GHz}$ WiFi. That can be employed in conjunction with using ESP AT firmware to give WiFi access to additional hosting Microcontrollers or even a self-assisted Microcontroller when coupled with an RTOS-based API. In this system, this ESP8266 module is used to accumulate the readings from different sensors and pass them to the remote server using internet-enabled services, as well as the graphical illustration of the utilized ESP8266 module is mentioned in the following figure, Fig- 6 .

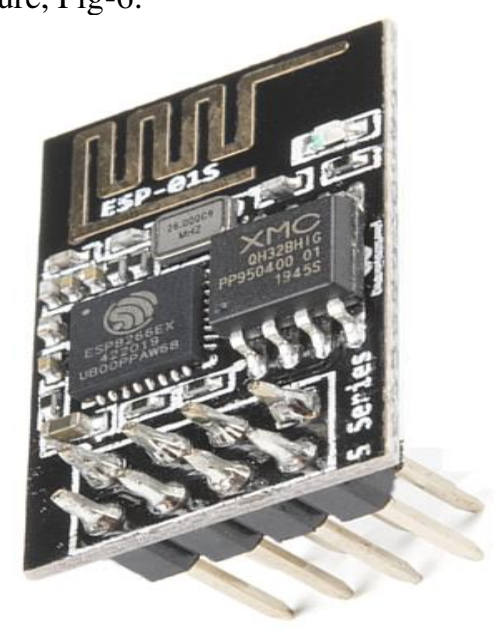

Fig. 6. ESP8266 Controller

The following figure, Fig-7 illustrates the logical block diagram of the proposed intelligent health monitoring kit called Smart Health Indicator (SHI), and the following algorithm illustrates the logical flow details of the proposed algorithm called Deviated Learning-based Health Analysis (DLHA). 


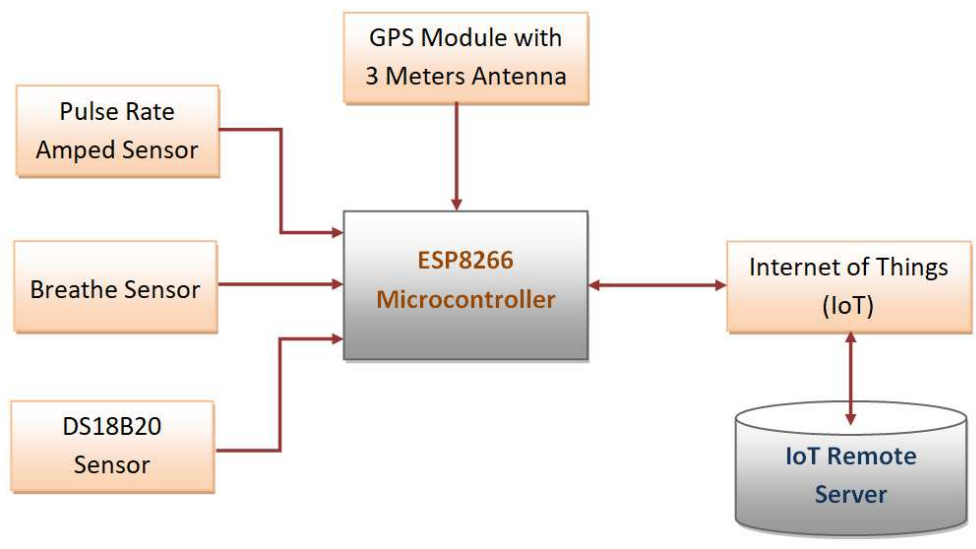

Fig. 7. Block Diagram

\section{Algorithm: Deviated Learning-based Health Analysis}

Input: Real-time Patient Health Record such as Heartbeat Level (H), Temperature Level (T), Breathe Level (B), Latitude and Longitude Values (L).

Output: Prediction and Health Record Maintenance.

Step-1: Initialize the required libraries into the implementation.

Step-2: Acquire the inputs $H, T, B$, and L. Assemble these inputs into the array with proper indexing.

Step-3: Load the dataset model created for the previous input values.

Step-4: Define the threshold ratio of the sensor values such as $H, T$, and $B$.

Step-5: Cross-validate the present values of H, T, and B to the loaded dataset threshold ratio.

Step-6: If the threshold level is within a range, the details are stored into the server using the respective IPs.

Step-7: If the threshold level crosses the present sensor values, then the details of the respective caretaker and doctor will be retrieved.

Step-8: Immediately send an alert to the respective individuals regarding an emergency with correct location details $(L)$. 
Step-9: Identify the accuracy ratio of prediction for accurate positive and false negative ratios.

Step-10: Return the prediction accuracy ratio of the proposed algorithm to the user's view.

\section{$4 \quad$ Results and Discussions}

The previous section specifically linked research papers that evaluate and continuously monitor health status, whereas the Internet of Things sends alert messages to physicians and related notifications to patient's caretakers when a condition deteriorates. This will not notify surrounding hospitals or physicians regarding the issue. However, this may be accomplished in this article and demonstrated through the conceptual framework model. All health care data and physician status can be refreshed and saved on a serverside periodically. An innovative IoT-enabled controller called ESP8266 is utilized since it is affordable and provides patients with much more effective legitimate information. The controller serves as a remote server and is significantly less expensive than a traditional server. The proposed emulation represents the difference between these two servers and denotes the physical separation between two places. Overall, this paper structure gives information about how patients can protect their lives without relying on others to come to them and take care of them when they are in the terrible medical condition possible. Everybody has their existence, and that no one concern more about anybody as they do of their difficulties, this Internet of Things technology assists patients in saving their lives by regularly updating.

The proposed smart device called Smart Health Indicator (SHI) is placed into the real-time environment and accumulates the details for continuous 10 days, and reports the results accordingly. The proposed resulting scenarios are cross-validated with the conventional learning algorithms called CNN and SVM to prove the efficiency of the proposed approach called DLHA. The following figure, Fig-8, illustrates the temperature analysis accuracy ratio of the proposed approach in contrast cross-validated with the conventional logics such as CNN and SVM. 


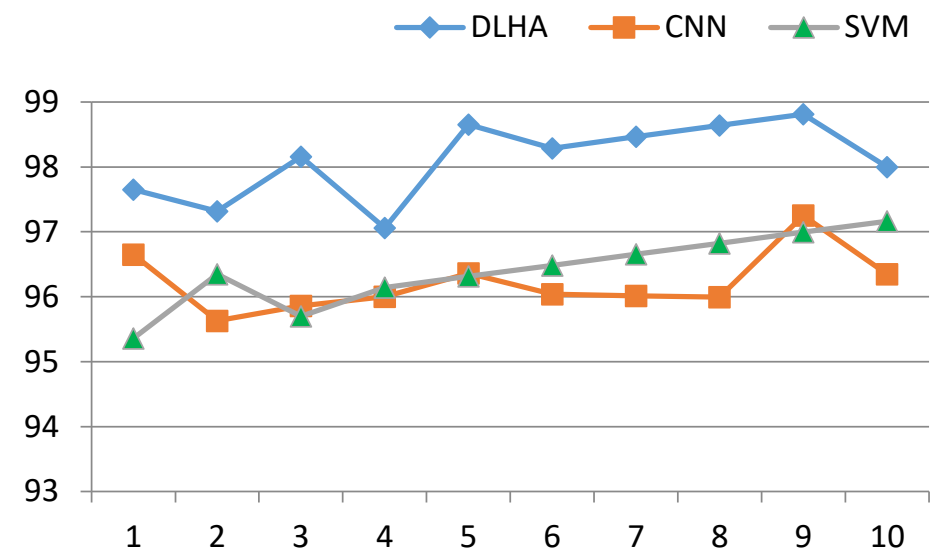

Fig. 8. Temperature Measurement

The following figure, Fig-9 illustrates the Data Collection accuracy ratio of the proposed approach in contrast that is cross-validated with the conventional logics such as CNN's and SVM's data accumulation accuracy level.

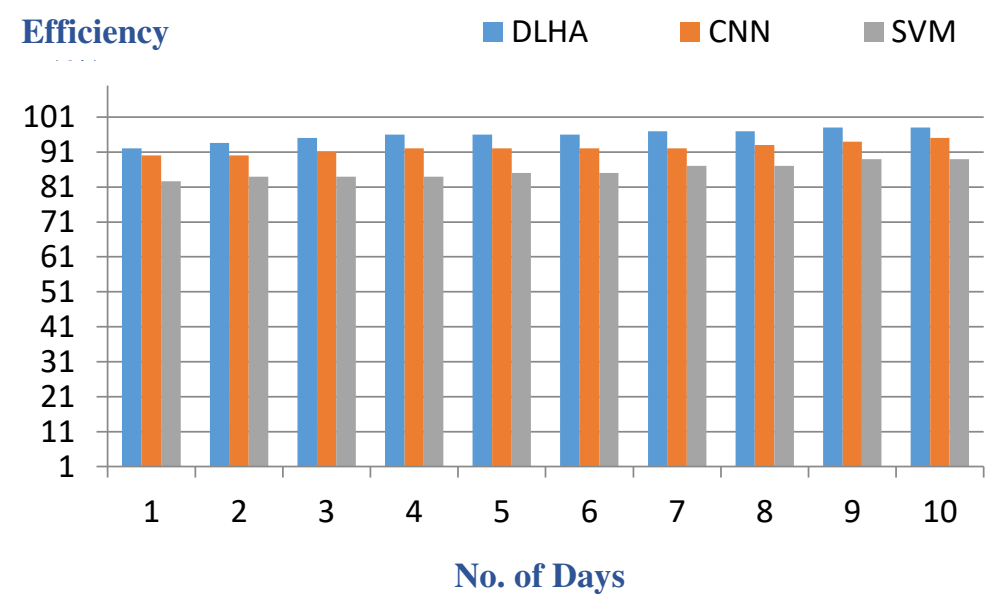

Fig. 9. Data Accumulation Efficiency

The following figure, Fig-10, illustrates the Data Loss ratio over the accumulation period of the proposed approach in contrast that is cross-validated with the conventional logics such as CNN's and SVM's loss ratio. 


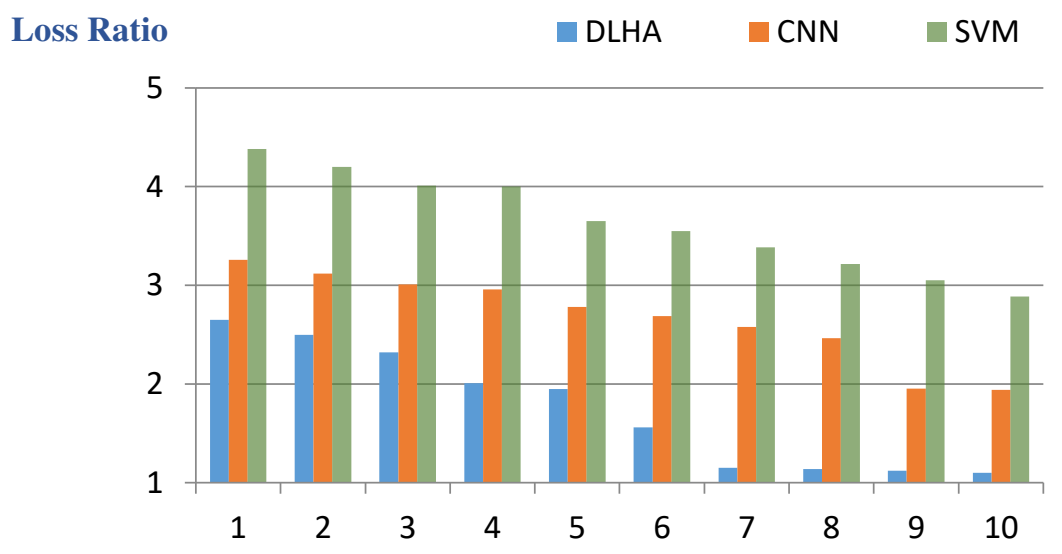

No. of Days

Fig. 10. Data Loss Ratio

The following table, Table-1, illustrates the proposed approach DLHA accuracy ratio with the other conventional algorithms such as CNN and Support Vector Machine. The proposed algorithm of DLHA provides $97.9 \%$ accuracy, CNN provides $91.49 \%$ accuracy [9], and SVM provides $77.63 \%$ [10]. The following figure, Fig-11, illustrates the proposed approach prediction accuracy ratio in contrast cross-validated with the conventional logics such as CNN's and SVM's prediction accuracy ratio.

Table 1. Accuracy Estimations

\begin{tabular}{|l|l|l|}
\hline S.No. & Algorithm & Accuracy \\
\hline 1 & DLHA & $97.9 \%$ \\
\hline 2 & CNN & $91.49 \%$ \\
\hline 3 & SVM & $77.63 \%$ \\
\hline
\end{tabular}




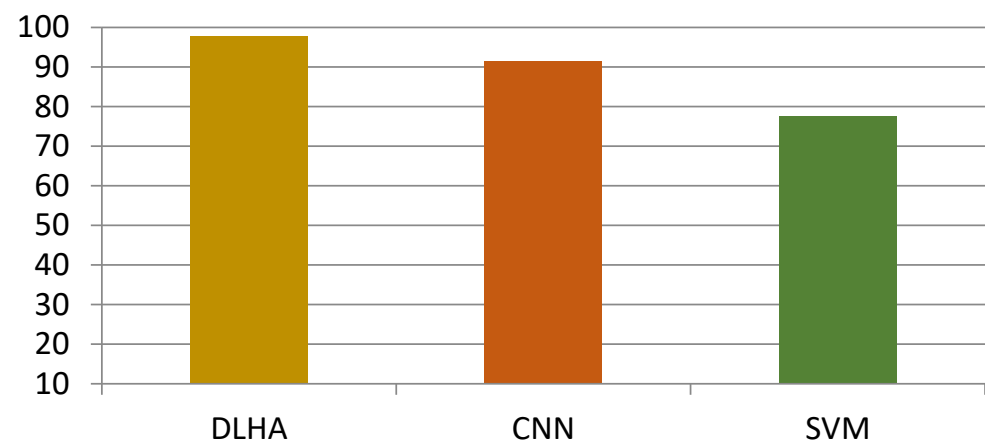

Algo-

Fig. 11. Accuracy Level Comparison

\section{$5 \quad$ Conclusion and Future Work}

The purpose of this research paper is to offer an Internet of Things-based intelligent healthcare monitoring system for actual patient monitoring via medical diagnostics computation. The proposed approach DLHA improves the utility of intelligent sensors based collection of data, consolidation, and management by providing relatively low impulses that can extend the lifetime of the entire network. Health care governance is a critical component of both individual and societal health. Automation and digitalizing health care services essentially reduce the burden on individuals and the medical community. By reduction, that means reduced spending, effort, and time, and the possibility of a monitoring system from the remote end for patients about long-term and constant monitoring of multiple biological data. In addition, these methods are easier to use, and individuals can rely on them. These systems monitor and detect signals in real-time and then communicate them to the appropriate person for quick aid, whether a doctor or a family member. This type of support is now possible, owing to advances in the realm of communication, most notably the internet. Internet of Things-based health care methods can provide individuals with the highest-quality healthcare services possible, allowing for early detection of various ailments and thus a reduction in mortality rates.

As previously said, there are numerous advantages to IoT-enabled healthcare systems. As mentioned in the paper, this was made possible by the numerous development boards manufactured by numerous manufacturers. These boards' primary objective is to interconnect thousands and multitudes of people and devices for the benefit of the public. These Internet of Things-based products are both efficient and inexpensive. Additionally, these boards equipped with AI can deliver a preliminary diagnosis to the patient. As a result, this will undoubtedly improve the quality of medical services offered to society while also lowering expenses. 
In the future, the work can further be enhanced using adding some time synchronization algorithms to reduce the overall processing time to train the model with multiple records over a single instance. This will add significance to the proposed approach of DLHA in a transparent manner.

\section{Acknowledgment:}

We sincerely acknowledge the Hindusthan College of Engineering and Technology, Coimbatore, Tamil Nadu for supporting this study through the Hindusthan College of Engineering and Technology, Coimbatore, Tamil Nadu Researchers Supporting Project.

\section{Declaration:}

Ethics Approval and Consent to Participate: No participation of humans takes place in this implementation process

Human and Animal Rights: No violation of Human and Animal Rights is involved.

Funding: No funding is involved in this work.

Conflict of Interest: Conflict of Interest is not applicable in this work.

Authorship contributions: There is no authorship contribution

Acknowledgment: We sincerely acknowledge the Hindusthan College of Engineering and Technology, Coimbatore, Tamil Nadu for supporting this study through the Hindusthan College of Engineering and Technology, Coimbatore, Tamil Nadu Researchers Supporting Project.

\section{Conflict of Interest}

Manuscript title: An Experimental Evaluation of Hybrid Learning Methodology based Internet of Things Assisted Health Care Monitoring System

Corresponding Author Name: Dr Mahaveerakannan R, Hindusthan College of Engineering and Technology, Coimbatore, Tamil Nadu

We have no conflict of interest to declare. This statement is to certify that all authors have seen and approved that the manuscript is submitted. 
On behalf of all Co-Authors, the corresponding author shall bear full responsibility for the submission.

All the authors sign this statement to indicate agreement that the above information is accurate and correct.

The corresponding author shall have to complete and submit this form to the Editorial Office. I certify that there is no actual or potential conflict of interest with this article.

Signature

Dr Mahaveerakannan R

\section{References}

[1] Bansal.M and Gandhi.B, "IoT Based Smart Health Care System using CNT electrodes (for continuous ECG monitoring)", International Conference on Computing, Communication and Automation, 2017.

[2] The top 10 causes of death in India, Hindustan-Times, Online: https://www.hindustantimes.com/health/the-top-10-causes-of-deathin-india/story-

1FLxCFVHmF7svw2RKCl70K.html, 2017.

[3] Bansal.M et al., "The genre of applications requiring longterm and continuous monitoring of ECG signals", International Conference on Innovations in Information, Embedded and Communication Systems, 2018.

[4] Elanthiraiyan.P et al., "Smart Medicine and Physical Health System Using IoT", International Journal of Computer Science and Mobile Computing, 2015.

[5] Holler.J et al., "From Machine-to-Machine to the Internet of Things: Introduction to a New Age of Intelligence", Amsterdam, The Netherlands: Elsevier, 2014.

[6] Mukhopadhyay et al., "Internet of Things: Challenges and Opportunities. In: Mukhopadhyay S. (eds) Internet of Things. Smart Sensors, Measurement and Instrumentation". Springer, 2014.

[7] Riazul et al., "The Internet of Things for Health Care: A Comprehensive Survey", IEEE Access, 2015.

[8] Vasanth.K et al., "Creating solutions for health through technology innovation", Texas Instruments. Online: http://www.ti.com/lit/wp/sszy006/sszy006.pdf, 2018.

[9] Walaa N. Ismail, Mohammad Mehedi Hassan, Hessah A. Alsalamah and Giancarlo Fortino, "CNN-Based Health Model for Regular Health Factors Analysis in Internet-of-Medical Things Environment", IEEE Access, 2020.

[10] Youn-Jung Son et al., "Application of Support Vector Machine for Prediction of Medication Adherence in Heart Failure Patients", Healthc Inform Res. 2010 Dec; 16(4): 253-259. 
[11] Inderpreet Singh and Deepak Kumar, "Improving IOT Based Architecture of Healthcare System", International Conference on Information Systems and Computer Networks, 2019.

[12] Abdulatif Alabdulatif, Ibrahim Khalil, Xun Yi and Mohsen Guizani, "Secure Edge of Things for Smart Healthcare Surveillance Framework", IEEE Access, 2019.

\section{Author Biography}

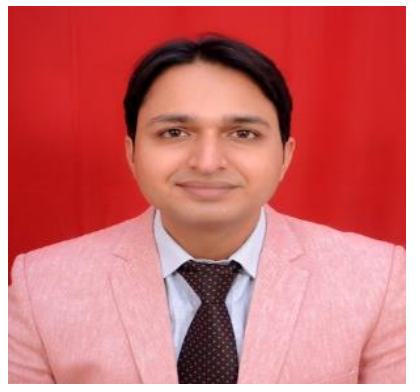

Dr. Kamal Upreti is currently working as an Associate Professor in the Department of Information Technology, Dr. Akhilesh Das Gupta Institute of Technology \& Management, Delhi. He completed his B. Tech (Hons), M. Tech (Gold Medalist), PGDM(Executive), and Ph.D. in Computer Science \& Engineering.

He has published many patents, books, magazine issues, and research papers in various reputed international conferences and journals. His areas of research interest include Machine Learning, Wireless Networking, Embedded systems, and Cloud Computing. In addition, he has been chaired many sessions in National and International Conferences across India.

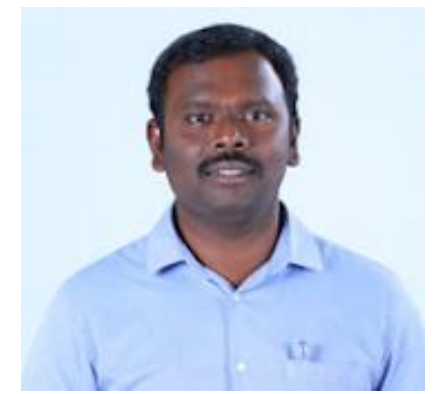

Dr. Mahaveerakannan $\mathbf{R}$ is currently working as an Assistant Professor in the Department of Information Technology, Hindusthan College of Engineering and Technology, Coimbatore. He completed his B. Tech (Information Technology) from A.V.C College of Engineering and Technology, Mayiladuthurai, M. Tech (Mainframe Technology) from the Anna University of Technology, Coimbatore, Ph. D (Information Technology) from St. Peters Institute for Higher Education and Research, Avadi, Chennai. He has published three patents and research papers in various reputed international conferences and journals. His areas of research interest include Machine Learning, Wireless Networking. 


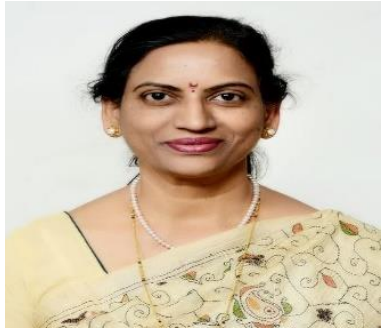

Professor Dr. Raut Ranjana Dinkar graduated from Govt. College of Engineering Pune, in 1986 with distinction. She did her M.E. and Ph.D. in Electronics Engineering. She has an Industrial Experience of 45 days at Hindustan Antibiotics, Pimpri, Pune, and a Total Teaching Experience of 35 years at various renowned institutions and P. G. Dept. of Applied Electronics, Faculty of Science and Technology. Sant Gadge Baba Amravati University, Amravati (M.S.) India. Her areas of research interest include Research Biomedical Electronics, Machine Intelligence, Artificial Intelligence, Image Processing, Mobile Computing, I.O.T., and Precise Agriculture Electronics.

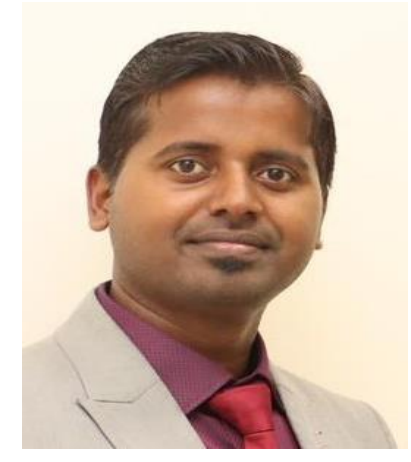

Dr. Sudhanshu Maurya is Post Doctoral Researcher at the School of Computer \& Communication Engineering, Universiti Malaysia Perlis (UniMAP), under the guidance of YBhg. Prof. Ir. Ts. Dr. R Badlishah Ahmad, ViceChancellor, Universiti Malaysia Perlis (UniMAP), Kangar, Perlis, Ministry of Education, Malaysia.

He has been designated as Assistant Professor in School of Computing, Graphic Era Hill University, Bhimtal Campus, Uttarakhand, India. He has contributed as a Research Fellow at NIT Surat on the project "CAIM Discretization of Columnar DBMS" and also at Jawaharlal Nehru University, New Delhi on the project "Natural Language Processing".

His area of research is dedicated to IoT, Cloud Computing, Mobile Cloud Computing, and Artificial Intelligence. Currently, he has 1 Edited Book*, 2 Course Books, 2 international Patents (Granted), 5 Indian patents, and more than 50 research papers indexed in SCOPUS, Web of Science, ACM, IEEE \& UGC Approved Journals. He has attended several conferences, FDPs, and workshops in various renowned Institutions like IIT Kanpur, IIT Kharagpur, IIT Guwahati, IIT Patna, IIT Bhilai, SV NIT Surat, JNU New Delhi, Central University of Rajasthan, and many others. 

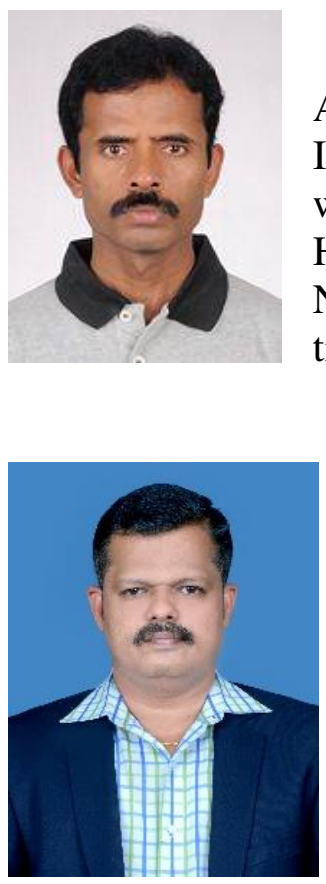

Y. Venkatramana Reddy received the M. Tech from Andhra University, Visakhapatnam, Andhra Pradesh, India. Ph D from N I T Tiruchirappalli. He is currently working as Associate Professor at Anurag University, Hyderabad, India. His main research interests include Network Security, Cryptography, Distributed Computing, and Cloud Computing.

Dr. Thangadurai. $\mathbf{N}$ is working as a Professor and Associate Director - Research in Sankalchand Patel University, Visnagar, Gujarat, India. He has obtained his Ph.D. Degree in Wireless Sensor Networks from Bharathiar University, Coimbatore. He has obtained his Bachelor's Degree in Electronics and Communication Engineering from Coimbatore Institute of Technology and Master's Degree in Applied Electronics from Mohamed Sathak Engineering College under Anna University and MBA from Alagappa University. He has more than 18 years of experience in Teaching, Research and Administration. He has authored/co-authored 167 research papers in both International and National Journals and Conferences. He has supervised 64 numbers of undergraduate and postgraduate students for their project completion and guiding $8 \mathrm{Ph}$.D. scholars now. 4 research scholars have been awarded for their Ph.D. under his guidance. He received sponsored research project grant from VGST, ISRO, MSME, DST and AICTE. He has also filed 26 Indian patents into his credit and that are published. Also 2 design patents filed. He got granted one Australian patent into his credit. He also authored/co-authored 7 books \& 6 Book Chapters into his credit. His research interests are Wireless Communication, Satellite Communications, Mobile Adhoc and Wireless Sensor Networks, Biomedical Engineering, Embedded Systems, Optical Communication and Navigation Systems. He is also a Life member of following professional bodies like ISCA, ISTE, IAENG, and IACSIT. Also, he is a Fellow of IETE and OSI and Senior Member of IEEE. He has received many awards like Best Young Scientist Award2018, Award for Research Publications from VGST 2016-17, Bharat Excellence Award -2017, Outstanding Researcher Award - 2016. 